\title{
Reinforced variation and selection
}

\author{
ALLEN NEURINGER \\ Reed College, Portland, Oregon
}

\begin{abstract}
Long-Evans rats were reinforced for generating variable sequences of four left $(\mathrm{L})$ and right (R) leverpress responses. If the current sequence, for example, LLRR, differed from each of the preceding five sequences, then a food pellet was provided. Otherwise, there was a brief time-out. After the rats were generating a variety of sequences, one sequence was concurrently reinforced whenever it occurred, that is, an "always-reinforced" contingency was superimposed. The frequency of that sequence increased significantly from the variable baseline (Experiment 1 ). The "difficulty" of the always-reinforced sequence influenced the extent to which its frequency increased-"easy" sequences increased greatly, but "difficult" ones increased not at all (Experiment 2, and a replication with pigeons in Experiment 3). However, when reinforcement for baseline variability was systematically decreased while a difficult sequence was always reinforced, that sequence slowly emerged and attained relatively high levels (Experiments 4 and 5). Thus, a technique for training even highly improbable, or difficult-to-learn, behaviors is provided by concurrent reinforcement of variation and selection.
\end{abstract}

The conditioning of operant responses has been compared to evolutionary processes in that basic to both are principles of variation and selection (e.g., Skinner, 1966, 1981,1984; Staddon, 1983). Operant responses are commonly hypothesized to be selected, or strengthened, by reinforcing events contingent upon such responses (Skinner, 1938). Behavioral variations, on the other hand, are most commonly hypothesized to result from the withholding of reinforcement, or response extinction (e.g., Antonitis, 1951; Eckerman \& Lanson, 1969; Lachter \& Corey, 1982; Schwartz, 1982). Thus, selection and variation of operants are thought to result from two sides of reinforcement, presentation and omission. There are, of course, other causes for behavioral variations, such as environmental noise (Bandura, 1982), drugs, and injury to the nervous system (Devenport, 1983). In addition to these factors, reinforcers contingent upon behavioral variation may also be important. That is, contingent reinforcement may not only select responses, but also cause them to vary.

That behavioral variability can be reinforced has been shown in a number of cases. For example, when improbable behaviors were followed by food to porpoises, they learned to generate highly unusual flips and turns (Pryor, Haag, \& O'Reilly, 1969). Similarly, when pigeons' "least frequent" interresponse times were reinforced, the pigeons learned to respond in a way similar to a random generator (Blough, 1966). A third example is that reinforcement of relatively infrequent sequences of leverpresses in rats or

\footnotetext{
This research was supported by NSF Grant BNS-8707992. The author expresses appreciation to Elizabeth McElroy and Ralph Huntley for assistance in conducting the research and analyzing the data and to Gene Olson for caring for the animals. Helpful critical comments were provided by Armando Machado and Cheryl Voss. Correspondence should be addressed to A. Neuringer, Department of Psychology, Reed College, Portland, OR 97202 (e-mail: aneuring@reed.edu.)
}

keypresses in pigeons led to high levels of sequence variability (Machado, 1989; Morgan \& Neuringer, 1990; Neuringer, 1991; Page \& Neuringer, 1985). These demonstrations that behavioral variability can be directly reinforced suggest the following hypothesis: the same reinforcers that maintain behavioral variations may also select and strengthen instances from the variable substrate. The present experiments tested this hypothesis.

Under the basic procedure, each trial consisted of four responses by rats to left (L) and right (R) levers. Baseline variations were maintained by requiring that the current sequence of four responses differ from each of the previous five sequences for reinforcement, a lag 5 variability schedule. If the current sequence repeated any one of the previous five, a brief time-out followed. Thus, for example, an LRRR sequence would be followed by reinforcement if exactly that sequence had not occurred during any of the previous 5 trials and by a time-out if it repeated any of the previous 5 . These contingencies have been shown to maintain high levels of sequence variability in rats and pigeons (Machado, 1989; Morgan \& Neuringer, 1990; Neuringer, 1991; Page \& Neuringer, 1985). To study whether particular sequences from this variable baseline could be selectively strengthened and weakened, Experiment 1 superimposed two contingencies. One of the 16 possible sequences was reinforced whenever it occurred, whether or not the variability contingency had been met, and was referred to as the always sequence-it was always reinforced. For comparison, a second sequence was never reinforced, again whether or not the variability contingency had been met, and was consequently referred to as the never sequence. In all other respects, the lag 5 variability contingencies were continued. The importance of whether individual sequences could be strengthened and weakened from a baseline of variations is threefold. First, the experiment asks whether 
reinforcement can concurrently serve to engender behavioral variability and select for and against particular instances. Second, this procedure may provide a method to analyze and compare the contributions of reinforcement to variation and selection. Third, concurrent reinforcement of baseline variation and individual instances may provide a means to train relatively intractable behaviors by maintaining overall response strength at levels necessary for selection to occur.

\section{EXPERIMENT 1 Selection From a Variable Substrate}

\section{Method}

Subjects. The subjects were 6 male Long-Evans rats, approximately 14 months old at the beginning of the experiment, that had previously been reinforced for generating variable sequences of leverpresses (Neuringer, 1991). They were deprived of food for $21 \mathrm{~h}$ before each session, following which they received $2 \mathrm{~h}$ free access to food. Water was freely available in their home cages, and they were maintained under a 12:12-h light:dark cycle, with experimental sessions occurring during the light phase, generally 5 days per week.

Apparatus. Six modified Gerbrands operant chambers $(27 \times 29 \times$ $30 \mathrm{~cm}$ ) had ceiling, back, and front walls constructed of Plexiglas and sidewalls of metal. One sidewall contained two response levers, $5 \mathrm{~cm}$ above the floor and $9 \mathrm{~cm}$ apart; centered between them was a pellet tray, $3 \mathrm{~cm}$ above the floor, into which were dispensed $45-\mathrm{mg}$ Noyes food pellets. The opposite sidewall contained three pigeon keys (not used in this experiment). On top of the ceiling was an 11-W clear houselight. A speaker, located behind the wall containing the levers, provided auditory stimuli. The chambers were housed in sound- and light-attenuating outer boxes containing one-way mirrors for observation. Macintosh computers were interfaced through Metaresearch Benchtop modules to the experimental chambers, and programs were written in TrueBasic.

Procedure. The procedure consisted of a baseline and three experimental phases.

Baseline. Each trial contained exactly four effective responses. If the current four-response sequence of $L$ and $R$ responses differed from each of the last 5 sequences, a lag 5 variability contingency, the trial terminated with reinforcement, a 45-mg Noyes food pellet (Morgan \& Neuringer, 1990; Neuringer, 1991; Page \& Neuringer, 1985). The pellet was presented immediately following the fourth response in the trial. If the current sequence repeated any one of the previous 5 sequences, the last response was followed by a 5 -sec time-out, during which the chamber was dark and an intermittent $600-\mathrm{Hz}, .05-\mathrm{sec}$ tone sounded. Responses to either lever during the time-out reset it, that is, $5 \mathrm{sec}$ of no responding was required before the houselight was reilluminated and effective responses were again possible. A 0.5 -sec interresponse interval (IRI) followed each of the first three responses in the trial, during which the chamber was dark. The IRI was also reset by responses. Each session lasted until 200 trials were emitted or $1 \mathrm{~h}$, whichever occurred first. Five baseline sessions were provided.

Experimental Phase 1: Always and never. Baseline contingencies were maintained exactly as just described, while concurrently one of the 16 possible sequences (given four responses on $L$ and $R$ levers, there are $2^{4}=16$ different possible sequences) was designated always and another sequence was designated never. Whenever the always sequence occurred, the trial ended with reinforcement, whether or not the above-described variability contingency had been met. Whenever the never sequence occurred, the trial ended with a timeout, again whether or not the variability contingency had been met. The two sequences were chosen individually for each rat such that they had approximately equal baseline probabilities approximating
.10. Thus, both always and never sequences occurred at intermediate levels during the baseline phase. Once the two sequences were chosen for each rat, a coin flip determined which of the two would be always and which never. For Subject 2223, for example, LLRR was always reinforced and RLLL was never reinforced. In brief, then, superimposed on the lag 5 variability contingencies were always and never contingencies. Reinforcement for always consisted of two food pellets, as compared to the one pellet contingent upon meeting the variability requirement; this difference may have been necessary in order for the subjects to discriminate between the always sequence and all others. Time-out for never was the same as under the variability contingencies, a resettable 5-sec time-out. The always (A) and never $(\mathrm{N})$ sequences for each of the 6 subjects were: $A=L R R R, N=R R R L ; A=L L R R, N=R L L L ; A=R L R R$, $\mathrm{N}=$ RRLL; $\mathrm{A}=\mathrm{LLRR}, \mathrm{N}=\mathrm{RLLL} ; \mathrm{A}=\mathrm{LRRR}, \mathrm{N}=\mathrm{LLLL} ;$ and $A=L L R R, N=R R R R$. After approximately 8 sessions, the always reinforcement was increased to four pellets, and approximately 8 additional sessions were provided until frequencies of always and never sequences stabilized across sessions, as assessed by visual inspection. Four food pellets continued to be provided for the always sequence (and one pellet for meeting the variability contingency) during the remaining phases of the experiment.

Experimental Phase 2: Always and never reversed. Contingencies were identical to those just described except that the always and never sequences were reversed. Thus, for 1 subject, $A=L R R R$ and $N=R R R L$ in Phase 1 , and these contingencies were now reversed such that $A=R R R L$ and $N=L R R R$. Fourteen to 32 sessions were provided, until frequencies of always and never sequences stabilized, as assessed by visual inspection.

Experimental Phase 3: Return to original contingencies. Contingencies were again reversed, so that they were identical to the original always and never conditions. Eight to 27 sessions were provided, again until each animal reached stable emissions of always and never sequences.

\section{Results}

Figure 1 shows percentages of always and never sequences (number of always and never sequences, respec-

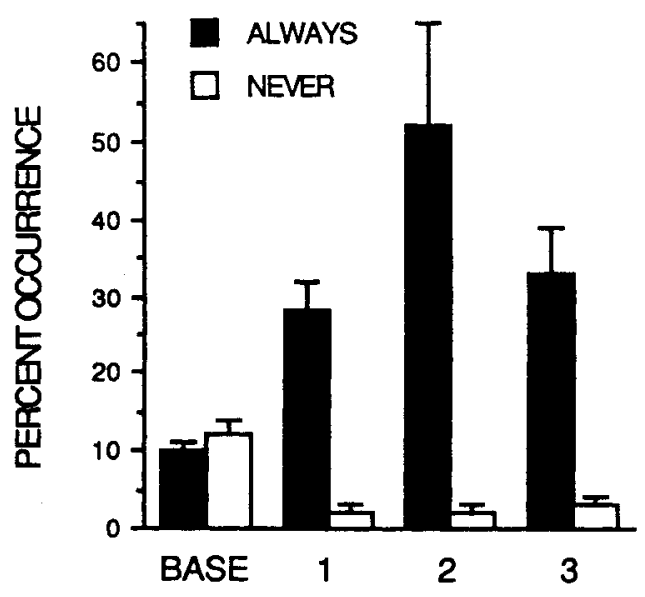

Figure 1. Percentage of occurrence of sequences that were always followed by food reinforcement (dark bars) and sequences that were never followed by food (open bars). During baseline (base), all sequences were reinforced only if they met a variability contingency. During Phase 1 , the always and never contingencies were superimposed on the variability baseline. Phase 2 was a reversal of the Phase 1 contingencies - the always sequence became never, and the never became always-- and Phase 3 again reversed the contingencies. Data are averages of 6 rats; error bars indicate standard errors. 
tively, divided by total sequences per session and multiplied by 100) averaged over the last 5 sessions for each of the four phases and over the 6 subjects. The bars labeled "base" show frequencies during the baseline variability phase, with the sequence that became always in the next phase shown by the dark bar and the sequence that became never shown by the open bar. Responding during the baseline phase was distributed across the 16 sequences, with average $U$ value, an index of sequence variability (Page \& Neuringer, 1985), equal to .81 (with 0 equal to minimum variability and 1.00 equal to maximum) and average probability of reinforcement (reinforced trials divided by total trials) equal to approximately .50 . During Phase 1, where the always sequence received continuous reinforcement and the never sequence was extinguished, the always sequence (dark bar) increased in frequency, while the never sequence (open bar) decreased. This same pattern of results was obtained when the always and never sequences were reversed in Phase 2 (always becoming never, and vice versa) and again when the Phase 1 conditions were reinstated in Phase 3. Statistical analyses confirmed these observations, with a $4 \times 2$ (phase, including baseline $\times$ contingency) analysis of variance (ANOVA) showing significant phase $[F(3,15)=$ $4.088, p<.05]$, contingency $[F(1,5)=52.176, p<$ $.001]$, and interaction $[F(3,15)=6.835, p<.005]$ effects. Most importantly, a contrast among means analysis showed that when the always and never contingencies were operating, the always sequences increased in frequency relative to baseline $[F(1,5)=38.84, p<.005]$ and the never sequences decreased $[F(1,5)=37.38, p<$ $.005]$. During baseline, the two sequences did not differ significantly, whereas during the three remaining phases of the experiment, the always sequences were significantly more likely to occur than were the never sequences $(p<$ .05 ). Finally, as might be expected from the preceding results, average $U$ values decreased to .64 during the three experimental phases, and average probability of reinforcement increased to .71 . The basic finding is that from a baseline of reinforced variations, sequences were selectively strengthened and weakened by presenting and withholding similar reinforcements.

\section{EXPERIMENT 2 \\ Selection as a Function of Sequence Difficulty}

Experiment 1 showed that, given a baseline of reinforced variations, when one sequence was continually reinforced, its frequency increased significantly, and when a second sequence was never reinforced, its frequency decreased significantly. Experiment 2 asked whether the "difficulty" of the selected sequences influenced this variation-and-selection effect. Three sequences were compared, one hypothesized to be "easy," LLLL, another to be of "intermediate difficulty," LRRR, and a third to be "difficult," LLLR. (Counting in binary, and arbitrarily assigning $L=0$ and $R=1$, these are sequences 0,7 , and 1 , respectively.) Previous research had shown that when reinforcement was provided independently of sequence variability in a two-lever chamber, rats were most likely to respond on a single lever and that LRRR was more likely to occur than LLLR (e.g., McElroy \& Neuringer, 1990; Morgan \& Neuringer, 1990; Neuringer \& Huntley, 1992). We therefore hypothesized that LLLL would most readily be strengthened by an always contingency and that LRRR would more readily be strengthened than LLLR. To simplify the procedure, only the always contingency was superimposed (a never contingency was no longer provided). Furthermore, to examine whether reinforcement for always sequences had to differ quantitatively from baseline reinforcers, identical reinforcers followed successful meeting of the variability and always contingencies, namely, a single food pellet. The main questions, therefore, were these: Does selection from a variable substrate depend upon the "difficulty" of the sequence, and can identical reinforcers engender both variation and selection?

\section{Method}

Subjects and Apparatus. Four of the 6 rats from Experiment 1 served immediately following that experiment. The apparatus was the same as in Experiment 1.

Procedure. The baseline variability procedure was identical to that in Experiment 1, with variable sequences continuing to be reinforced under a lag 5 variability procedure. The following changes in procedure were then instituted: (1) The difficulty of the always sequence was varied, (2) a single food pellet was contingent upon the always sequence as well as the variability criterion, (3) one always sequence was used for all subjects, (4) the never contingency was omitted, and (5) baseline phases followed each always phase. Following the initial baseline ( 25 sessions), an LLLL always contingency was concurrently provided for all rats. In addition to the reinforcement of variable sequences, LLLL was reinforced whenever it occurred. Reinforcement for the always sequence was a single pellet, identical to that given when the variability contingency was met. After 7 sessions, baseline was reintroduced for 5 sessions. A second always sequence was then concurrently provided, RLLL, and this was maintained for 35 sessions. A return to baseline ( 12 sessions) was then followed by the LLLR always sequence, and this contingency was maintained for 23 sessions, with the experiment ending with 11 sessions of baseline. In all cases, the number of sessions depended upon visually assessed stability of responding.

\section{Results}

The solid bars in Figure 2 show percentages of each of the three sequences (LLLL, LRRR, and RRRL) when they were "always" reinforced, and the striped bars represent the percentages of these same sequences during the preceding and following baseline phases, when only the lag 5 variability contingency was in effect. Averages over the last five sessions of each phase are shown. When the always contingency was applied to the easy LLLL sequence, it increased to more than $85 \%$. When the intermediate-difficulty LRRR sequence was selectively reinforced, it increased to approximately $50 \%$. However, reinforcement of the most difficult sequence, LLLR, had no effect-its percentage of occurrence remained at baseline level. To simplify statistical analysis, the two baseline values surrounding each always phase were averaged, 


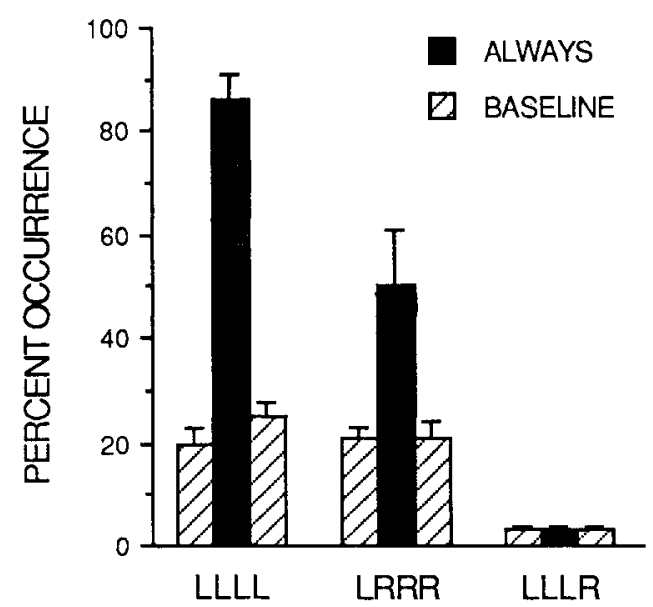

Figure 2. Percentage of occurrence of LLLL, LRRR, and LLLR sequences (dark bars), when these were always followed by food reinforcement, and their levels under baseline conditions (striped bars), when only the variability contingencies were in effect. The subjects were 4 rats; error bars indicate standard errors.

and a $3 \times 2$ ANOVA was performed using sequence difficulty $(0,1$, and 7$)$ and contingency (baseline vs. always) as the main factors. Statistically significant effects of both sequence difficulty and contingency were observed $[F(2,6)=50.900, p<.0005$, and $F(1,3)=86.230$, $p<.005$, respectively]. Of most importance, a significant contingency $\times$ sequence interaction was also obtained $[F(2,6)=32.527, p<.001]$, with always sequences significantly more likely during the always phases than during baseline for Sequences $0[F(1,3)=222.94, p<$ $.001]$ and $7[F(1,3)=10.42, p<.05]$, but not for Sequence $1[F(1,3)=.360$, n.s.]. Furthermore, in the presence of their respective always contingencies, Sequence 0 occurred more frequently than Sequence 7 , which, in turn, occurred more frequently than Sequence 1 ( $p<.01$ in both cases). The main conclusion is that sequence difficulty influenced the extent to which reinforcement strengthened a selected sequence. Also, identical reinforcements sufficed to maintain baseline variations and to select instances from the baseline.

\section{EXPERIMENT 3 \\ Selection as a Function of Sequence Difficulty in Pigeons}

To test the generality of the variation-and-selection effects observed in Experiments 1 and 2, this experiment essentially replicated the procedures of Experiment 2, with pigeons rather than rats serving as subjects.

\section{Method}

Subjects. Three homing pigeons of undetermined gender, born and raised in the Reed College Animal Colony, were maintained at $85 \%$ of their free-feeding body weights. They had previously been trained to peck response keys for food reward.
Apparatus. Three Gerbrands operant chambers, measuring $28 \mathrm{~cm}$ wide $\times 29 \mathrm{~cm}$ high $\times 27 \mathrm{~cm}$ deep, contained three pigeon keys spaced equidistantly $7 \mathrm{~cm}$ apart on the front wall, each of which could be transilluminated with white light. Only the left and middle keys were used in the current experiment. Directly below the middle key was a $6 \times 4.5 \mathrm{~cm}$ opening to a grain hopper that provided mixed pigeon grain as reinforcer. An overhead 11-W white bulb provided houselight. Each chamber was connected to a Macintosh computer.

Procedure. The procedure was similar to that in Experiment 2. Each trial consisted of four responses to the two keys, a response causing a 0.5 -sec darkening of both keys (IRI), with the houselight remaining on. If the current sequence of four responses differed from each of the previous 5 sequences (lag 5), a reinforcement resulted, consisting of 2-4 sec access to mixed grain (the duration of reinforcement depending upon the weight gain of each bird). Repetitions of any one of the previous 5 sequences resulted in a 5 -sec time-out, during which the chamber was completely dark. Both IRI and time-out were reset by responses. Sessions terminated after the 60th reinforcement. These baseline contingencies lasted for 19-23 sessions, until stability was reached. There followed three phases analogous to those in Experiment 2. First, an RRRR always contingency (Sequence 15 in binary) was superimposed for 6-8 sessions, following which there was a return to baseline ( 6 sessions), 33-37 sessions with an LRRR always sequence (Sequence 7), 12-15 additional baseline sessions, 23-27 sessions with an LLLR always sequence (Sequence 1), and a final 10-13 sessions of baseline.

\section{Results}

The results essentially replicated with pigeons those obtained in Experiment 2 with rats. Figure 3 shows that the always contingency again had the greatest strengthening effect for the easy sequence, an intermediate effect for the intermediate-difficulty sequence, and no effect for the most difficult sequence. The same statistical analyses as in Experiment 2 again showed that both sequence and contingency effects were statistically significant $[F(2,4)=$ $72.903, p<.001$, and $[F(1,2)=246.857, p<.005$, respectively] and that the interaction between sequence

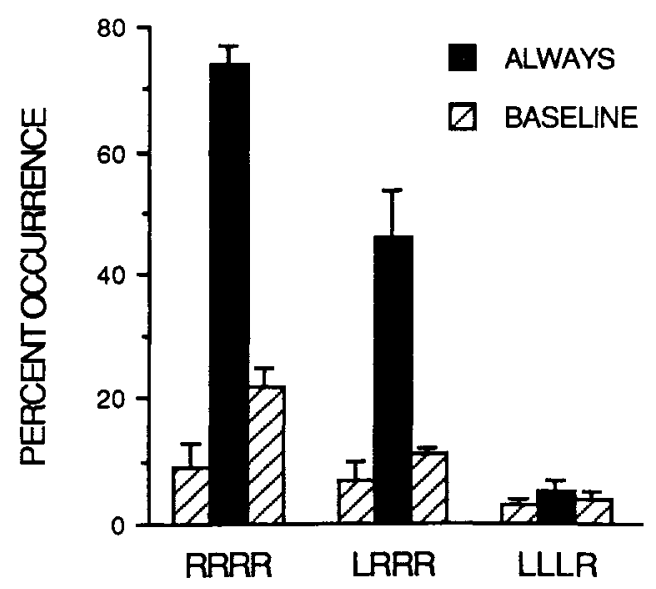

Figure 3. Percentage of occurrence of RRRR, LRRR, and LLLR sequences (dark bars), when these were always followed by food reinforcement, and their levels under baseline conditions (striped bars), when only the variability contingencies were in effect. The subjects were 3 pigeons; error bars indicate standard errors. 
and contingency was also significant $[F(2,4)=17.59$, $p<.05$ ], with the always sequence more likely to occur when it was selectively reinforced during the always phase than during baseline for Sequence $15,[F(1,2)=272.76$, $p<.01]$, marginally greater for Sequence $7[F(1,2)=$ $14.54, p<.10]$, but not more likely to occur for Sequence $1[F(1,2)=2.29$, n.s.] Sequence 15 occurred more frequently than Sequence 7 , which, in turn, occurred more frequently than Sequence 1 ( $p<.05$ in both cases). Thus, pigeons as well as rats showed that difficulty of a sequence influences the selectively strengthening effects of an always contingency and that the same reinforcer can engender baseline variability and strengthen particular instances.

\section{EXPERIMENT 4 Fading of Reinforcement for Variability}

Experiments 2 and 3 might be taken to indicate that selection from a variable baseline occurs only for behaviors that are relatively likely, or easy. Many alternative hypotheses are possible, however, such as that emission of the always sequence depends upon its difficulty versus that of behaving variably or upon the relative frequencies of reinforcement for the always sequence versus variable sequences (see, e.g., Neuringer, 1992). The current experiment progressively decreased the frequency of reinforcement for baseline variability while maintaining an always contingency for a most improbable sequence. The basic question was whether a difficult sequence can be strengthened by an always contingency if reinforcement for variability is progressively faded. Fading was accomplished by reinforcing variable sequences only intermittently, according to a variable-interval (VI) schedule, where reinforcers were programmed to occur randomly in time. Reinforcement for sequence variations became less and less frequent across sessions.

\section{Method}

Subjects. The subjects were 8 female Long-Evans rats, approximately 9 months old, that had previously served in a variability experiment where four-response trials were reinforced under lag 4 contingencies (Neuringer \& Huntley, 1992). They were housed and maintained as in Experiment 1.

Apparatus. The experimental chambers differed somewhat from those used in Experiments 1 and 2. Four modified Gerbrands operant chambers, $27.5 \mathrm{~cm}$ long $\times 26.5 \mathrm{~cm}$ deep $\times 29 \mathrm{~cm}$ high, with Plexiglas ceiling and front and back walls, and metal left and right walls, were housed in sound- and light-attenuating outer boxes. An 11-W white houselight was above the ceiling of the chamber, and the left wall contained three levers, $5 \mathrm{~cm}$ long $\times 1 \mathrm{~cm}$ thick, spaced equidistantly $(3.5 \mathrm{~cm}$ between the levers and $2 \mathrm{~cm}$ between the outer levers and the chamber walls) and $9 \mathrm{~cm}$ above the floor. Only the two outer levers were active, with transluscent white stimulus lights $10 \mathrm{~cm}$ above these levers illuminated when responses were effective. As in the previous studies, 45-mg Noyes food pellets again served as reinforcers, but they now fell into a recessed compartment directly under the middle lever.

Procedure. The variability contingencies were similar to those in Experiments 1 and 2, except for the following. First, a VI schedule was added to the variability criterion such that reinforcement was possible only after the current interval had elapsed: reinforcement was given for the first trial to meet the variability criterion following the timing out of the current interval. Second, the current sequence had to differ from the last three sequences to meet the variability criterion, or lag 3 , rather than the lag 5 used in the first three experiments.

At the beginning of each trial, the houselight and stimulus lights over the levers were illuminated. A response caused the stimulus light above the lever to darken and a $2000-\mathrm{Hz}$ tone to sound for $0.15 \mathrm{sec}$. Each trial ended with one of three outcomes: (1) If the trial did not meet the lag 3 variability criterion, the chamber was dark and silent for $1 \mathrm{sec}$. Leverpresses were not counted (i.e, the reset contingency used in Experiments 1 and 2 was not employed). When $1 \mathrm{sec}$ had elapsed, the houselight and the two stimulus lights came on, and the next trial began. (2) If the trial did meet the lag 3 variability criterion, but the current VI had not elapsed, the chamber again went dark for $1 \mathrm{sec}$, but now a warbling tone (a 0.05 $\mathrm{sec}, 600-\mathrm{Hz}$ tone alternated with a $0.05-\mathrm{sec}, 3300-\mathrm{Hz}$ tone) repeated for the 1 -sec interval. At the end of this interval, during which leverpresses were not counted, the warbling tone stopped and the houselight and the two stimulus lights were illuminated, signaling the beginning of the next trial. (3) If the trial met the lag 3 variability criterion and the current VI interval had elapsed, the stimuli were the same as in Outcome 2, and, in addition, a Noyes food pellet was presented at the end of the 1-sec interval, after which the lights came back on to initiate another trial. A reinforced trial caused a new interval in the VI sequence to begin. Nine baseline sessions were provided under lag 3 variability, with 5-sec VI contingencies. Sessions lasted for $1 \mathrm{~h}$.

Baseline frequencies of the 16 possible sequences were compared, and it was found that LLRL (Sequence 13) was the least likely sequence to occur on average across the 8 subjects. Beginning with the 10th session, therefore, LLRL was designated as always, its occurrence always followed by reinforcement, whether or not the variability contingency had been met or the VI had elapsed. For all other sequences, the VI 5-sec variability contingencies were concurrently operative. Reinforcers for always sequences were identical to the reinforcers for variation, that is, a single Noyes food pellet. Whenever LLRL was emitted and reinforced, the current variable interval was reset. The always plus VI 5 -sec variability contingencies were maintained for 6 sessions, after which the VI value increased to $10 \mathrm{sec}$ for 2 sessions and by $10 \mathrm{sec}$ every $2 \mathrm{nd}$ session thereafter, with a few minor exceptions (e.g., 4 sessions were given at VI $70 \mathrm{sec}$ ). Following the $61 \mathrm{st}$ session, the VI increased by $10 \mathrm{sec}$ after every session (rather than every 2 nd session) until VI $500 \mathrm{sec}$, and that value was maintained for 9 additional sessions. Thus, LLRL was always reinforced, while the frequency of reinforcement for variable sequences was systematically reduced. The variability contingency was then removed altogether for 7 additional sessions so that reinforcement depended solely upon the LLRL always sequence. Finally, the always contingency was removed, and for 21 sessions the rats were reinforced under a VI $30-\mathrm{sec}$, lag 3 variability contingency. During these last 21 sessions of return to baseline conditions, LLRL was reinforced only if it met the variability contingencies.

\section{Results}

Overall frequencies of responding are of interest as well as frequencies of the always sequence, and these data are therefore presented separately. Figure 4A shows the number of LLRL sequences emitted per session as a function of five-session blocks throughout the experiment. (Where number of sessions was not divisible by five, one of the blocks was increased in size, e.g., the first baseline block was five sessions and the second was six sessions.) The 
(A)

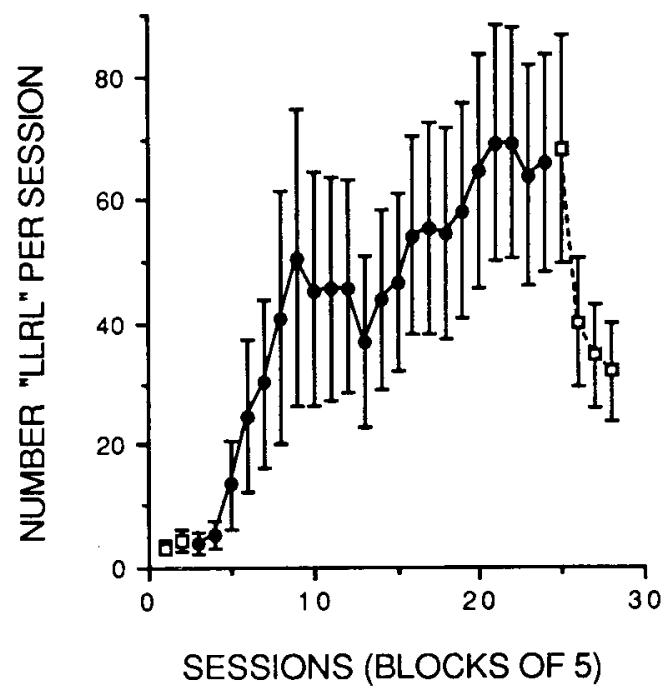

(B)

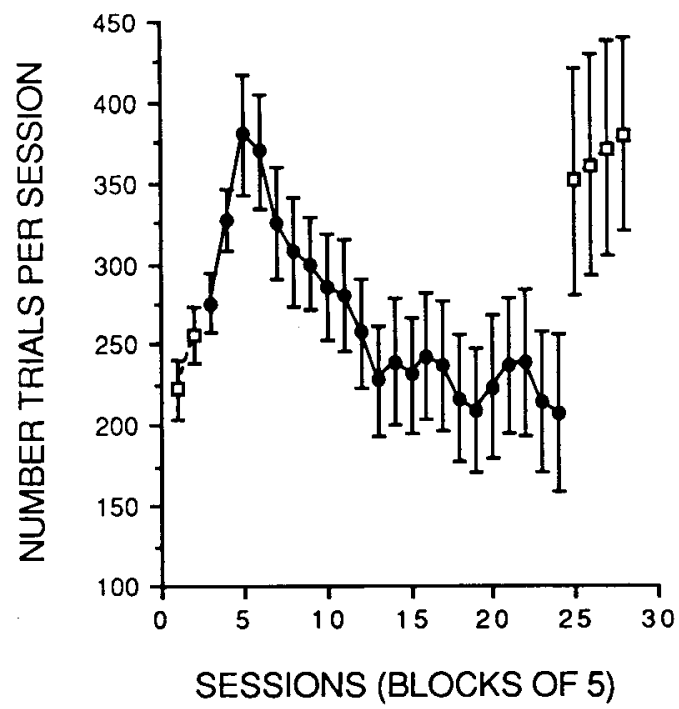

Figure 4. (A) Number of LLRL sequences emitted per session over five-session blocks. (B) Total number of trials emitted per session. Open squares indicate baseline, when only variability contingencies were in effect. Closed circles show concurrent reinforcement of all instances of the LLRL sequence. Data are averaged over 8 rats; standard errors are shown by the error bars.

first two open points are from baseline, before the always contingency was instituted, where there were fewer than five occurrences of LLRL per session. By the end of the always phase (dark circles), more than 60 LLRL sequences were emitted on average per session, a statistically significant increase $[F(7,23)=6.261, p<.0001]$. When the always contingency was removed, as shown by the last four open points in Figure 4, frequencies of LLRL decreased. Thus, a high strength of LLRL, the sequence least likely to occur during the baseline sessions, was attained via a process of always reinforcing that sequence and slowly fading reinforcement for variability; its continued high strength depended upon its continued reinforcement.

Figure 4B shows the total number of trials emitted per session. The initial increase in trials per session was correlated with the subjects' learning to emit the LLRL sequence. The decrease after the fifth block of sessions was correlated with the decrease in reinforcement for variation as well as the increase in frequency of reinforcement for LLRL. When the always contingency was removed during the last phase (open squares) and a more frequent VI 30-sec was reintroduced, the number of sequences per session again increased.

In addition to the low baseline frequency of the LLRL sequence, its difficulty was indicated by two additional observations. First, 1 of the 8 subjects never learned to emit it, thus accounting for the large standard errors shown in Figure 4. Second, even though the frequency of the always sequence increased significantly, by the end of the experiment the LLRL sequence was occurring only $30 \%$ of the time, that is, always divided by total sequences was approximately $30 \%$, as can be seen by comparing Figures $4 \mathrm{~A}$ and $4 \mathrm{~B}$.

\section{EXPERIMENT 5 \\ Fading Reinforcement for Variability and Control}

Experiment 4 showed that an improbable sequence was increased in strength when it was always reinforced, while reinforcement for variability was slowly faded. Part of the explanation for this finding may be that the baseline variability contingency assured a necessary level of variations from which the always sequence eventually could be selected. In addition, the variability contingency may have maintained overall responding at sufficiently high strength for the always reinforcement to make contact with the behavior. However, the subjects had had prior experience with variability contingencies, which may have contributed in unknown ways to the Experiment 4 findings, and other aspects of the Experiment 4 manipulations may also have contributed. Experiment 5, therefore, employed two groups of experimentally naive rats, one of which, the experimental group, experienced fading of variability reinforcement as in Experiment 4, while the other, or control, group, was not reinforced for baseline variations. The basic goal of the experiment was again to test the hypothesis that fading concurrent reinforcement for behavioral variations enabled the selective strengthening of an improbable behavior.

\section{Method}

Subjects. The subjects were 10 experimentally naive Long-Evans female retired breeders that were approximately 9 months old at the beginning of the experiment. They were maintained as described in Experiment 1.

Apparatus. The apparatus was identical to that used in Experiment 4, except that five chambers were used.

Procedure. The rats were shaped to press $R$ and $L$ levers and then put on a fixed-ratio schedule of four leverpresses for 1 session, followed by a lag 3 variability schedule for 3 sessions. Two groups were then randomly constituted, consisting of 5 rats each, 
with an RRLR (Sequence 2) always contingency in effect for both groups. Throughout the remainder of the experiment, whenever the RRLR sequence was emitted, it was reinforced with a pellet. Contingencies for the variability group were similar to those in Experiment 4 , with a VI lag 3 variability schedule providing concurrent reinforcement, again a single pellet. The initial VI value was $10 \mathrm{sec}$, and this increased by $10 \mathrm{sec}$ every session. The control subjects had the same always contingency, but were never reinforced for behaving variably. Thus, the only way for the control subjects to obtain reinforcement was to emit the RRLR sequence. In brief, both groups were reinforced for every occurrence of RRLR, but the variability group was additionally reinforced for meeting the variability contingency, the latter contingency providing decreasing fre-

(A)

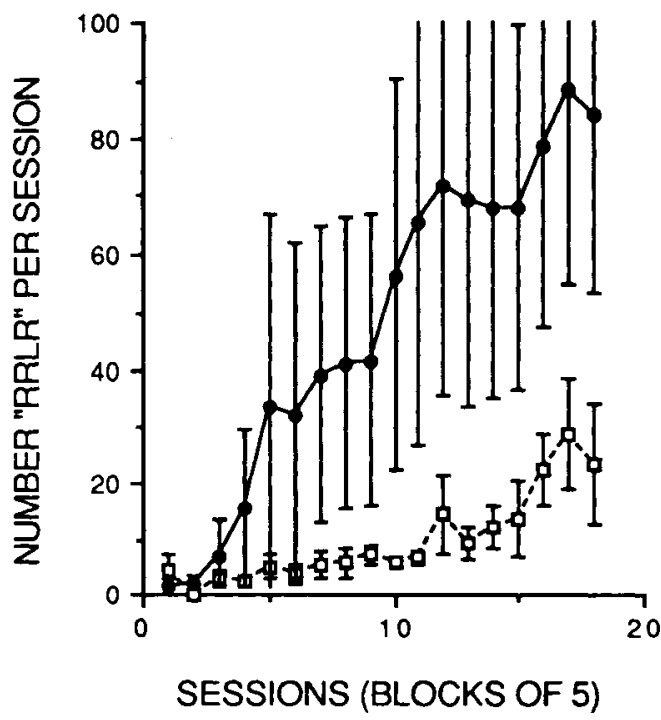

(B)

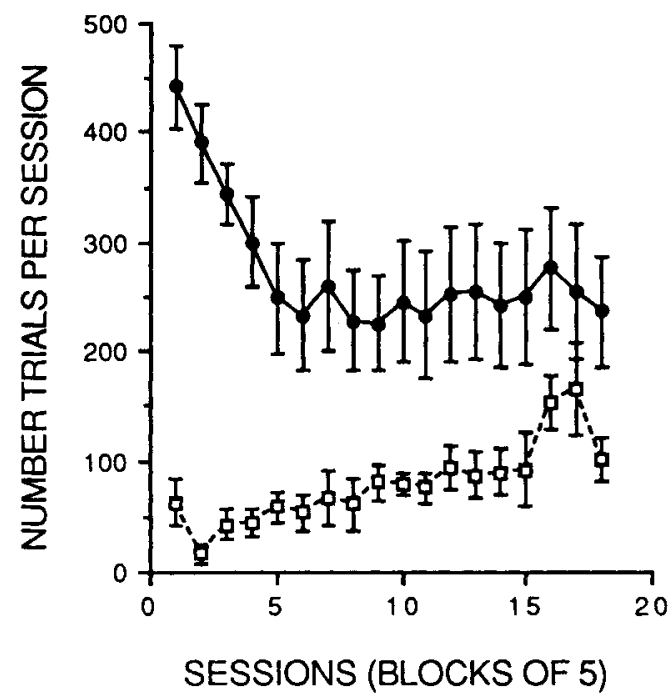

Figure 5. (A) Number of RRLR sequences emitted per session over five-session blocks. Open squares represent averages of 5 rats that were reinforced every time the RRLR sequence occurred. Closed circles show averages of 5 rats that were reinforced in the same manner and, in addition, occasionally reinforced for variable sequences. Error bars indicate standard errors. (B) Total number of trials emitted per session, with everything else as in (A). quencies of reinforcement across sessions. There were $88 \mathrm{llh}$ sessions, with the last session for the variability group containing a VI 880-sec contingency for sequence variations.

\section{Results}

Figure 5A shows the average number of always sequences emitted per session as a function of five-session blocks throughout the experiment (with the last block consisting of three sessions). During baseline, there were fewer than 5 RRLR sequences per session in both groups, but, by the end of the experiment, the variability group was averaging more than 80 such sequences per session, whereas the control group was averaging approximately 25 per session. Significant session effects $[F(17,136)=$ $5.038, p<.0001]$ and a group $\times$ session interaction $[F(17,136)=1.951, p<.05]$ were observed, and simple effects analyses showed that the variability group increased frequencies of RRLR sequences $[F(17,136)=$ $6.466, p<.001]$ whereas the control group did not $[F(17,136)=.523$, n.s. $]$ At the end of the experiment, the two groups differed significantly in their frequencies of always sequences $(p<.05)$

Figure 5B shows the average number of trials, or se: quences, per session. The variability group generally responded more than did the control group throughout the experiment $[F(1,8)=17.858, p<.005]$. A significant group $\times$ session interaction was obtained $[F(17,136)=$ $5.876, p<.0001]$, with the variability subjects' responses decreasing as the baseline VI increased, reaching asymptote at approximately 250 sequences per session. The control group's responses increased slowly throughout the experiment, but never attained levels as high as the variability subjects'.

The main findings were that reinforcement of baseline variability was correlated with high levels of responding overall, and as this baseline reinforcement was faded, high levels of the always-reinforced sequence emerged. Thus, this experiment confirmed and extended the results of Experiment 4 . Further research must directly test whether fading is a necessary part of the procedure. A constant frequency of reinforcement for variability might have been as effective. Although constant reinforcement for variability in Experiments 2 and 3 did not facilitate a difficult always sequence, different frequencies of reinforcement for variation should be examined.

\section{DISCUSSION}

The main question was whether reinforcement can maintain baseline response variability while at the same time selectively strengthening particular instances. The answer appears to be yes. Previous studies have shown that when a sequence was reinforced only if it had not occurred during the last $n$ trials, high levels of sequence variability were generated (Cohen, Neuringer, \& Rhodes, 1990; Machado, 1989; McElroy \& Neuringer, 1990; Morgan \& Neuringer, 1990; Neuringer, 1991; Page \& Neuringer, 1985). This reinforcement-of-variability effect was confirmed in the baseline phase of Experiment 1, 
as well as the baseline phases of each of the other experiments. When, concurrent with this reinforced variability, a single sequence was always reinforced (whether or not it met the variability contingencies), that sequence emerged from the variable substrate and significantly increased in probability. At the same time, the probability of a second sequence, selected for extinction, decreased. Thus, the subjects were sensitive both to the reinforcement of variations and concurrently to the reinforcement and extinction of particular instances. Perhaps most surprising in these findings was the high sensitivity of the subjects to the strengthening and weakening of selected sequences from a variable substrate. The results are consistent with the hypothesis that reinforcement serves the dual functions of selecting instances and concurrently engendering variability (see also Neuringer, 1992).

In Experiment 1, baseline variability was maintained by a reinforcer consisting of a single pellet, whereas the sequence selected to be always reinforced was followed by four pellets with every occurrence. This difference in reinforcement quantity might have been important for the variation-and-selection effects observed. However, in Experiments 2-5, reinforcers were identical-one pellet (or access to grain by pigeons in Experiment 3) followed sequences that met the variability criterion, and one pellet (or access to grain) also followed the selected sequence. Again, reinforcement was found to subserve dual functions, concurrently generating baseline variations as well as strengthening instances. Thus, qualitatively and quantitatively identical reinforcers will maintain baseline variability and at the same time selectively strengthen particular instances.

Experiments 2 and 3 showed that the difficulty of the sequences correlated with susceptibility to selection from a variable substrate. Assessment of difficulty was based on observations of baseline frequencies in previous studies when sequence variability was not required for reinforcement. During the initial baseline phases of the current studies, the frequencies of the easiest sequences, LLLL in Experiment 2 and RRRR in Experiment 3, did not differ from that of the intermediate-difficulty LRRR sequence, presumably because of the reinforcement of baseline sequence variability. When the always contingency was applied, however, the easy LLLL and RRRR sequences increased in frequency more than did the intermediate-difficulty LRRR sequence, which, in turn, increased more than did the most difficult LLLR sequence. Note that Experiments 2 and 3 presented the easiest sequences first and the most difficult sequences last, thereby confounding difficulty with order of presentation; future research should therefore test the conclusions reached.

The reinforced variation-and-selection procedures employed in the present experiments may be useful in educational and training situations. Experiments 4 and 5 suggest that even improbable, or difficult, responses can be selectively strengthened. These were sequences whose baseline frequencies were the lowest observed. By reinforcing dif- ficult sequences while concurrently providing systematically decreasing reinforcement for variation, the improbable sequences increased significantly in frequency. Educators and trainers are often challenged by behaviors whose baseline frequencies are extremely low. A number of further studies would be relevant to the educational domain. First, the efficacy of fading reinforcement should be compared with that of maintaining a constant, possibly intermediate frequency of reinforcement for baseline variations. Second, high-strength baseline responding can be maintained through means other than reinforcement of variations, and these should be explored. Third, variation-and-selection procedures should be compared with others normally used to train improbable behaviors, for example, shaping, trial and error, imitation, and verbal instruction. It would be of particular interest to explore the extent to which normal shaping and training procedures contain within them implicit reinforcement of behavioral variations.

Behavioral variability has many causes: drugs and physiological states (Devenport, 1983), extinction of responding through withdrawal of reinforcement (Antonitis, 1951; Eckerman \& Lanson, 1969; Lachter \& Corey, 1982; Schwartz, 1982), increased demands for greater number of responses (Boren, Moerschbaecher, \& Whyte, 1978; Eckerman \& Vreeland, 1973; Ferraro \& Branch, 1968; Stebbins \& Lanson, 1962; Wong, 1977), random environmental events (Bandura, 1982), and so forth. Direct reinforcement of variability may have particularly beneficial effects. If a variable substrate is necessary for strengthening highly complex or unlikely responses, reinforcing this variability may maintain the desired class of behaviors at a strength sufficient for selection to occur. On the other hand, other sources of variability, such as extinction, response difficulty, or environmental noise may weaken the desired response class. Extinction-induced aggression, depression, and attempts to escape from the environment may be avoided through direct reinforcement of variability. Thus, reinforced variation and selection may be a uniquely useful method to strengthen behaviors that are normally difficult to train.

\section{REFERENCES}

Antonitis, J. J. (1951). Response variability in the white rat during conditioning, extinction and reconditioning. Journal of Experimental Psychology, 42, 273-281.

Bandura, A. (1982). The psychology of chance encounters and life paths. American Psychologist, 37, 747-755.

BLouGH, D. S. (1966). The reinforcement of least frequent interresponse times. Journal of the Experimental Analysis of Behavior, 9, 581-591.

Boren, J. J., Moerschbaecher, J. M., \& WhYTe, A. A. (1978). Variability of response location on fixed-ratio and fixed-interval schedules of reinforcement. Journal of the Experimental Analysis of Behavior, 30, 63-67.

Cohen, L., Neuringer, A., \& Rhodes, D. (1990). Effects of ethanol on reinforced variations and repetitions by rats under a multiple schedule. Journal of the Experimental Analysis of Behavior, 54, 1-12.

DEVENPORT, L. D. (1983). Spontaneous behavior: Inferences from neuroscience. In R. Mellgren (Ed.), Animal cognition and behavior (pp. 83-125). Amsterdam: North-Holland. 
ECKERMAN, D. A., \& LANSON, R. N. (1969). Variability of response location for pigeons responding under continuous reinforcement, intermittent reinforcement and extinction. Journal of the Experimental Analysis of Behavior, 12, 73-80.

ECKERMAN, D. A., \& VREELAND, R. (1973). Response variability for humans receiving continuous, intermittent, or no positive experimenter feedback. Bulletin of the Psychonomic Society, 2, 297-299.

Ferraro, D. P., \& Branch, K. H. (1968). Variability of response location during regular and partial reinforcement. Psychological Reports, 23, 1023-1031.

Lachter, G. D., \& Corey, J. R. (1982). Variability of the duration of an operant. Behavioral Analysis Letters, 2, 97-102.

MaCHADO, A. (1989). Operant conditioning of behavioral variability using a percentile reinforcement schedule. Journal of the Experimenta Analysis of Behavior, 52, 155-166.

McElroy, E., Neuringer, A. (1990). Effects of alcohol on reinforced repetitions and reinforced variations in rats. Psychopharmacology (Berlin), 102, 49-55.

Morgan, L., \& Neuringer, A. (1990). Behavioral variability as a function of response topography and reinforcement contingency. Animal Learning \& Behavior, 18, 257-263.

NEURINGER, A. (1991). Operant variability and repetition as functions of interresponse time. Journal of Experimental Psychology: Animal Behavior Processes, 17, 3-12.

Neuringer, A. (1992). Choosing to vary and repeat. Psychological Science, 3, 246-250.

NeURINGER, A., \& HuNTLEY, R. W. (1992). Reinforced variability in rats: Effects of gender, age and contingency. Physiology \& Behavior, 51, 145-149.
Page, S., \& Neuringer, A. (1985). Variability is an operant. Journal of Experimental Psychology: Animal Behavior Processes, 11, 429-452.

Pryor, K. W., HaAg, R., \& O'Reill.y, J. (1969). The creative porpoise: Training for novel behavior. Journal of the Experimental Analysis of Behavior, 12, 653-661.

SCHWARTZ, B. (1982). Failure to produce response variability with reinforcement. Joumal of the Experimental Analysis of Behavior, 37, 171-181.

Skinner, B. F. (1938). The behavior of organisms. New York: Appleton-Century-Crofts.

Skinner, B. F. (1966). Phylogeny and ontogeny of behavior. Science, 153, $1205-1213$.

SkINNER, B. F. (1981). Selection by consequences. Science, 213, $501-504$

SKINNER, B. F. (1984). The evolution of behavior. Journal of the Experimental Analysis of Behavior, 41, 217-221.

StAdDon, J. E. R. (1983). Adaptive behavior and leaming. Cambridge: Cambridge University Press.

Stebbins, W. C., LANSON, R. N. (1962). Response latency as a function of reinforcement schedule. Journal of the Experimental Analysis of Behavior, 5, 299-304.

WoNG, P. T. P. (1977). A behavioral field approach to instrumental learning in the rat: $I$. Partial reinforcement effect and sex differences. Animal Learning \& Behavior, 5, 5-13.

(Manuscript received March 27, 1992; revision accepted for publication July 28,1992 .) 